\title{
SSD detection on LIDT tested coated Fused Silica samples
}

\author{
Heidi Cattaneo ${ }^{1 *}$, Daniel Schachtler ${ }^{2}$, Roelene Botha $^{2}$, and Oliver Fähnle ${ }^{1}$ \\ ${ }^{1}$ OST Eastern Switzerland University of Applied Sciences, Institute for Microtechnology and Photonics, 9471 Buchs, Switzerland \\ ${ }^{2}$ RhySearch, Optical Coating Division, 9471 Buchs, Switzerland
}

\begin{abstract}
Material changes and Sub-Surface Damage (SSD) under Laser Induced Damage Threshold (LIDT) testing sites were investigated on 3 diverse single layer coated transparent fused silica samples to obtain additional information on damage precursors on these samples. As a detection method, photothermal deflection technique utilizing a resonant UV laser beam was used. The local variations in UV absorption and probe beam transmittance due to previous exposure to high fluence laser pulses were strongly dependent on the coating itself and on the laser energy used during the LIDT testing. Also the obtained LIDT values differ from coating to coating. Detected effects on the coatings and substrate surface beneath ranged from small surface dislocations to complete coating damage with material transformations. Additional absorbing damage precursors were found close to the damaged sites.
\end{abstract}

\section{Introduction}

Sub-Surface Damage (SSD) such as cracks, contamination and local inhomogeneities in material's optical properties can cause enhanced surface absorption and lead to electrical and light field intensification in the material $[1,2]$. These defects prone to act as initiators for laser-induced damage resulting in material's premature degradation [3, 4], which is relevant especially when using coated optical components in high-power laser applications. SSDs are residual defects that originate from manufacturing and handling steps and typically reside few $\mu \mathrm{m}$ below the polished substrate surface, but in brittle materials some cracks can extend even beyond $100 \mu \mathrm{m}$ deep in the bulk material [1].

SSDs are typically detected using microscopic techniques. Many of these methods are based on scattering related effects of SSDs and are sensitive to local structural and refractive index inhomogeneities. Small laser-induced damage precursors, however, are mostly of absorptive nature and need to be detected accordingly. Photothermal microscopy is a measurement method that relies on absorption related changes in the material's optothermal properties. The radiation from a resonant pump beam causes local temperature and refractive index variations which can then be measured by their effects, such as beam deflection and defocusing, on a nonresonant probe beam.

Since the invention of the Q-switch, the obtainable peak pulse power of lasers has increased dramatically. These so-called giant pulses easily damage the optical components. The laser induced damaged threshold (LIDT) has become an important quantity to qualify optical components and coatings for high-power laser applications. [5]
In this study, photothermal deflection (PTD) method is used to detect SSD and other material variations in coated fused silica samples that have been previously subjected to strong laser irradiation during LIDT testing. The goal of the study is to investigate different laser induced damage sites and gain knowledge on the correlation between SSD and the resulting material changes. As some material and wavelength combinations show higher density of damage precursors or reduced resistance to laser induced damage, it's of interest to better understand the nature of these precursors, in order to comprehend the root cause of the damage and to improve the coatings.

\section{Experimental methods}

\subsection{Samples}

P4 polished (rms roughness of about $3 \AA$ ) fused silica substrates were used for the LIDT measurements. The substrates were cleaned by a multistep procedure at cleanroom facilities prior to the coating. The investigated coatings were a single-layer coating of $3 \mathrm{QW} \mathrm{1)}$ at 1064 $\mathrm{nm}$ of $\mathrm{Ta}_{2} \mathrm{O}_{5}, 2$ ) at $1064 \mathrm{~nm}$ and 3 ) at $355 \mathrm{~nm}$ of $\mathrm{SiO}_{2}$. The coatings were produced with reactive magnetron sputtering at Evatec AG.

\subsection{Laser-induced threshold testing}

The LIDT measurements were performed using a Litron LPYG-450-100 diode-pumped laser. The LIDT was measured at $1064 \mathrm{~nm}$ and $355 \mathrm{~nm}$ for the $\mathrm{SiO}_{2}$ layers and $1064 \mathrm{~nm}$ for $\mathrm{Ta}_{2} \mathrm{O}_{5}$. The laser operated at a repetition rate of $100 \mathrm{~Hz}$ with a pulse duration of $11 \mathrm{~ns}$. The laser beam

\footnotetext{
* Corresponding author: heidi.cattaneo@,ost.ch
} 
was focused onto the sample surface and the different test sites where irradiated with 5000 pulses per site. The sample was positioned on a $x-y-z$ motorized stage which allowed the automated irradiation of each test site. The effective beam diameter at the sample surface was around $230 \mu \mathrm{m}$. The online damage detection during the measurement was done with a photodiode which detected scattered light on the damaged site. The final offline damage detection was performed with differential interference contrast (DIC) microscope images of each test site. At least 150 sites were irradiated with appropriately chosen laser fluences over the transition regime between $0 \%$ and $100 \%$ damage probability.

\subsection{SSD detection}

A sensitive PTD microscope operating in transmission scheme with collinear pump and probe beams has been utilized to investigate SSD and material changes in optical samples. A detailed description of the setup can be found in Ref. 6. As a pump source, a frequency tripled Qswitched Nd-laser (TRUMPF TruMark 6350) having maximum average output power of $5 \mathrm{~W}$ and operating at $20 \mathrm{kHz}$ repetition frequency was used. The average power was reduced approximately to $130 \mathrm{~mW}$ before entering the sample. Alternatively, an externally modulated (125 $\mathrm{Hz}$ ) UV Diode laser (Toptica IBEAM-SMART) operating at $375 \mathrm{~nm}$ with maximum cw output power of $70 \mathrm{~mW}$ was used as pump source for samples coated with $\mathrm{Ta}_{2} \mathrm{O}_{5}$. The probe source was an intensity-stabilized $\mathrm{HeNe}$ laser having $1 \mathrm{~mW}$ optical power and power stability of \pm 0.2 $\%$ over $8 \mathrm{~h}$. The pump and probe beams were focused with the same $40 \mathrm{~mm}$ EFL UV-VIS achromatic objective to approximately $10-20 \mu \mathrm{m}$ spots on the sample surface.

The UV-induced probe beam deflection was measured as AC variations occurring at the repetition rate of the pump laser in two orthogonal position signals of a position sensitive detector (New Focus 2901). The relative transmitted optical power of the probe beam was obtained with the same detector as well. To attain 2D transmission and PTD mappings, the sample was mounted on a XYZ translation platform and its surface was scanned across the beam foci using linear translation stages having sub- $\mu \mathrm{m}$ resolutions. Several $2 \mathrm{D}$ mappings were also measured inside the material at different depths from the front surface of the sample.

The PTD method is sensitive to the relative positions of the pump and probe beam foci on the sample surface. Maximum response was typically achieved when the beams were crossing each other with a small angle (max few degrees) and partially overlapping at the sample surface. The relative positions of the beam foci were first roughly adjusted using a laser beam profiler or knife edge -technique and finally, by maximizing the PTD-signals obtained from a reference sample (such as NBK7) showing larger intrinsic UV absorption.

\section{Results}

Three fused silica samples with different single-layer coatings $\left(\mathrm{Ta}_{2} \mathrm{O}_{5}\right.$ for $1064 \mathrm{~nm}, \mathrm{SiO}_{2}$ for 1064 and $355 \mathrm{~nm}$ ) were tested using both the LIDT and PTD methods described above. The PTD testing was conducted under the LIDT test sites that have at least been partially damaged during the testing.

\subsection{LIDT}

The investigation of the damages with DIC microscopy revealed that the damage sites look very different for the three different samples studied. For the $\mathrm{Ta}_{2} \mathrm{O}_{5}$ coated sample (LIDT at $1064 \mathrm{~nm}$ ), the damages look like the coating was disintegrated around the centre of irradiation. There is also an additional less dramatic change of the material around the perimeter of the main damage zone.

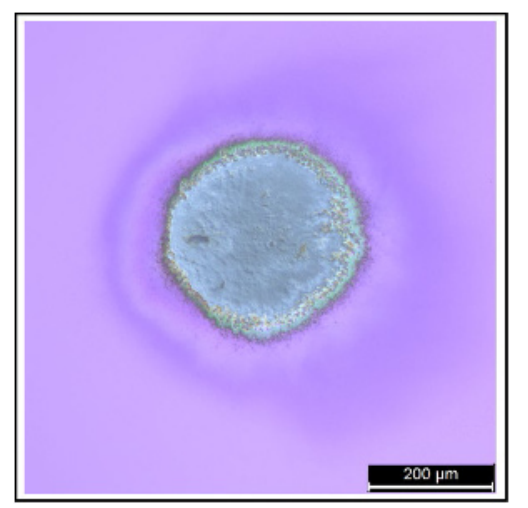

Fig. 1 DIC microscopy image of the investigated damage site (see also Fig. 4) of the $\mathrm{Ta}_{2} \mathrm{O}_{5}$ coated sample. The site was irradiated with an energy density of $8.8 \mathrm{Jcm}^{-2}$.

The damages of the $\mathrm{SiO}_{2}$ coated sample (LIDT at 1064 $\mathrm{nm}$ ) always appear around a damage precursor in form of a small defect at the centre. Around this precursor there is always quasi circular area which is visible with DIC microscopy. This circular area is called a plasma scald and it is caused by a laser-supported detonation wave, with its origin at the laser induced damage site [7].

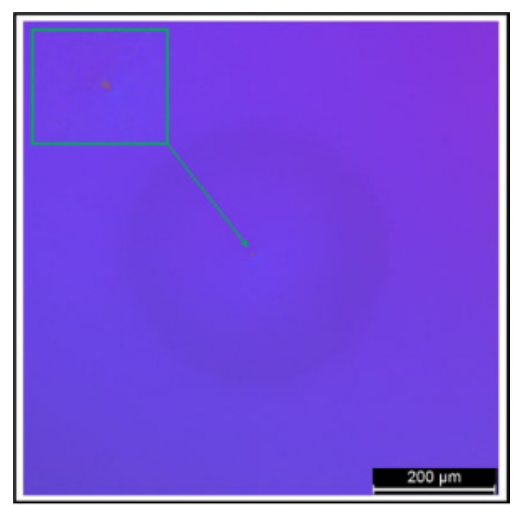

Fig. 2 DIC microscopy image of the investigated damage site (see also Fig. 5) of the $\mathrm{SiO}_{2}$ coated sample (1064 nm LIDT). The site was irradiated with an energy density of $130 \mathrm{Jcm}^{-2}$.

The damages of the $\mathrm{SiO}_{2}$ coated sample (LIDT at $355 \mathrm{~nm}$ ) appear as an agglomeration of small micron-sized defects. These defects in the laser irradiated area increase in density exponentially with increasing laser energy density. The damages have probably very small 
precursors which are not visible by light microscopy and show laser induced growth. This laser induced growth was also observed via scattered light detection.

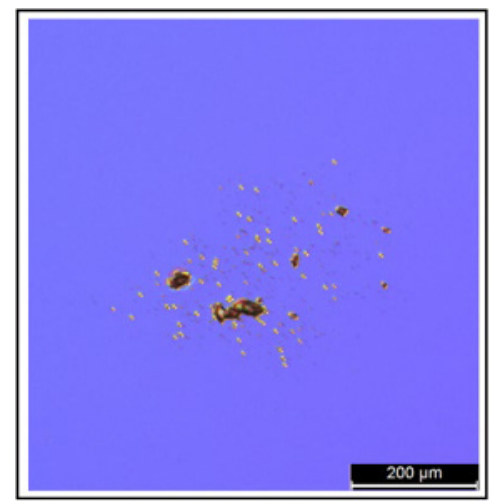

Fig. 3 DIC microscopy image of the investigated damage site of the $\mathrm{SiO}_{2}$ coated sample ( $355 \mathrm{~nm}$ LIDT). The site was irradiated with an energy density of $9.8 \mathrm{Jcm}^{-2}$.

\subsection{PTD}

The PTD response of the coated samples depended strongly on the coating itself and on the type of the laserinduced damage. The first sample $\left(\mathrm{Ta}_{2} \mathrm{O}_{5}\right.$ for $\left.1064 \mathrm{~nm}\right)$ exhibited relatively strong UV absorption and produced the highest PTD signals. In order to prevent further damage on the coating during the measurements, UV diode laser was used as a pump source. The damaged areas showed increased transmission $(+15-30 \%)$ of the probe beam and slightly higher PTD signals (up to x10) than the surrounding undamaged coating, indicating coating removal with material alterations on the substrate surface as the pump energy was not sufficient to produce PTD signal on pure fused silica. The edges of the damaged areas produced the most intense PTD peaks (up to $\mathrm{x} 50$ ). Around the damage areas, the increase in transmission of the probe beam (up to $20 \%$ ) was visible in form of a circular ring or a halo. There was no increase in PTD signal. The increase in transmission can be explained as surface dislocation. During PTD scanning, other small absorption centres were found as well on regions without prior damage. An example of transmission and PTD mappings performed simultaneously at the sample surface over one of the LIDT sites is shown in Figure 4.

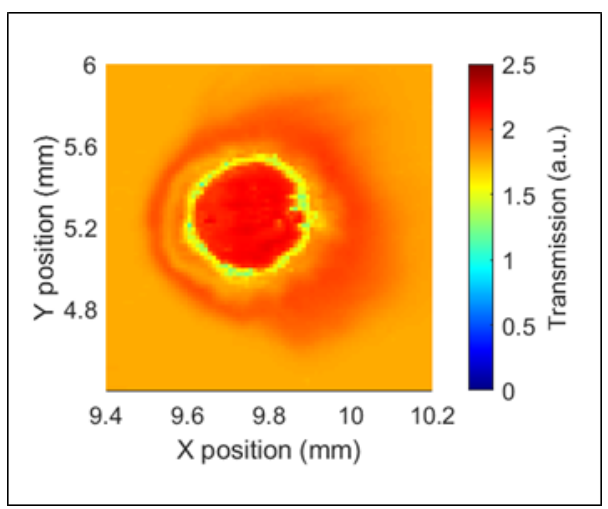

a)

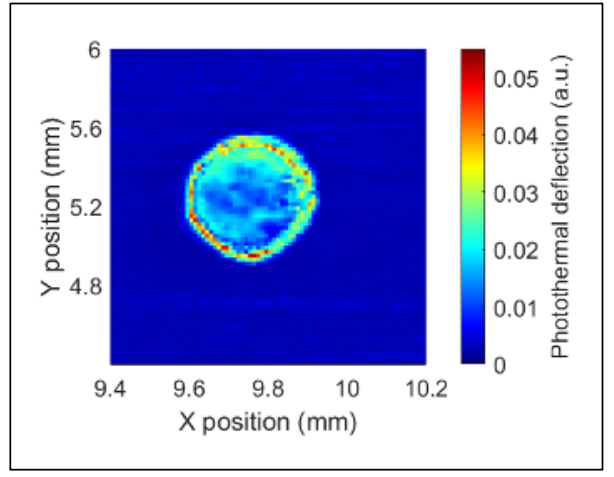

b)

Fig. 4. Example of a a) transmission and b) PTD mapping over a LIDT site in $\mathrm{Ta}_{2} \mathrm{O}_{5}$ coated fused silica sample. Scanning area $(800 \mu \mathrm{m})^{2}$, scanning step $10 \mu \mathrm{m}$, PSD gain 1 , and average pump power $18 \mathrm{~mW}$.

Few z-scans were performed as well and they revealed that LIDT testing has caused material deposits on the edges of the sites. Accordingly, maximum PTD signal was obtained few $100 \mu \mathrm{m}$ above the sample surface.

The second sample $\left(\mathrm{SiO}_{2}\right.$ for $\left.1064 \mathrm{~nm}\right)$ also produced faint interference fringes on probe beam transmission mappings over the LIDT sites indicating small surface dislocation. The magnitude of the dislocation increased with the radiation dosage used during the LIDT testing. For the UV absorption mapping, ns pulsed UV laser was used as a pump source to produce sufficient PTD signal as the $\mathrm{SiO}_{2}$ coating did not show higher UV absorption than the substrate itself. The measurements were performed $100-200 \mu \mathrm{m}$ below the surface to prevent further damage on the coatings due to the absorptioninduced local heating from the pulsed pump laser. The PTD mappings showed slightly increased signal at the edges of the plasma scalds and occasionally also at the precursor centre. For larger energy dosages, increased PTD signal was obtained on a larger area around the damage centre. An example of a PTD mapping performed $200 \mu \mathrm{m}$ below the sample surface over one of the LIDT sites is shown in Figure 5.

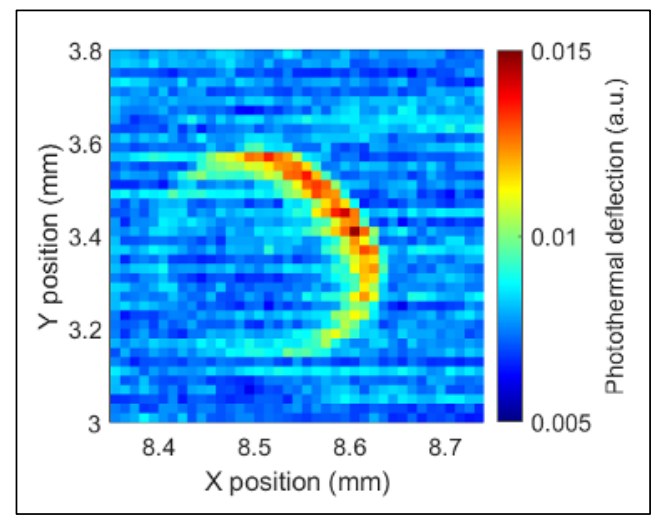

Fig. 5. Example of a PTD mapping over a LIDT site in $\mathrm{SiO}_{2}$ (for $1064 \mathrm{~nm})$ coated fused silica sample. Scanning area $(400 \mu \mathrm{m})^{2}$, scanning step $10 \mu \mathrm{m}$, PSD gain 10 , and average pump power $130 \mathrm{~mW}$. 
The coating itself was not damaged, but the increased PTD signal indicates possible delamination of the coating on the edges of the plasma scalds. According to the zscans there was no material deposit on the sample surface and the PTD signal was at maximum at the immediate close to the surface.

The other $\mathrm{SiO}_{2}$-coated samples showed increased transmission only around the completely damaged LIDT sites. No increase in UV-absorption was observed neither under the LIDT sites. The small damage markings were purely of scattering nature and caused locally reduced transmission and hence reduced photothermal deflection as well compared to the signals obtained from intact areas. Few small absorbing centres were incidentally found close to the irradiated sites.

\section{Conclusions}

Three different single-layer coatings were tested using LIDT S-on-1 with $\mathrm{S}=5000$ method. The obtained LIDT values varied between 3.5 and $80 \mathrm{Jcm}^{-2}$ and depended on the coating material and design wavelength. Similarly, the optical appearance of the damage sites differed greatly from coating to coating. This is also an indication that the underlying physical cause of the damages varies for different materials and photon energies. The appearance of the damages also partially depended on the energy dosage used during the testing, e.g. the visible defect density of the $\mathrm{SiO}_{2}$ coated samples tested at $355 \mathrm{~nm}$ increased exponentially with increasing energy density. The damage sites were additionally analysed using a combination of transmission and PTD measurements. Several lateral and in-depth scans were performed over the LIDT sites using UV pump and VIS probe laser beams. The laser induced effects on the coatings varied from small surface dislocations to complete coating disintegration with material alterations. Few additional small UV absorbing damage precursors were found close to the damage sites as well. No additional cracks nor other scattering type SSD were found below the coatings in the vicinity of the damage sites indicating that most of the small damage precursors were of absorbing nature. The damage precursors might be metallic or other absorbing particles coming from the coating process.

\section{Acknowledgements}

The authors thank Innosuisse - Swiss Innovation Agency for financial support (Project 39986.1 IP-ENG Coating technology for the high throughput, high yield production of dielectric optical interference filters for multispectral imaging). We wish to thank Evatec AG for providing the coatings.

\section{References}

1. J. Wang, Y., Li, J. Han, Q. Xu, Y. Guo, J. Eur. Opt. Soc.-Rapid 6, 1-16 (2011)

2. M. Chen, M. Li, C. An, L. Zhou, J. Cheng, Y. Xiao, W. Jiang, Jpn. J. Appl. Phys. 52, 1-8 (2013)
3. L. Hongjie, H. Li, W. Fengrui, Z. Xinda, Y. Xin, Z. Xiaoyan, S. Laixi, J. Xiaodong, S. Zhan, Z. Wanguo, Opt. Express 21, 12204-12217 (2013)

4. J. Neauport, L. Lamaignere, H. Bercegol, F. Pilon, J.-C. Birolleau, Opt. Express 13, 10163-10171 (2005)

5. Ristau, Detlev, ed. Laser-induced damage in optical materials (CRC Press, Hannover, 2014)

6. H. Cattaneo, R. Botha, C. Ziolek, Proc. SPIE 11352, 1135205-1 (2020)

7. X. Liu, Y. Zhao, D. Li, G. Hu, Y. Gao, Z. Fan, J. Shao, Appl. Opt. 50, 4226-4231 (2011) 